\title{
Listening to syncope
}

\author{
Guruprasad Sogunuru • Kishan Vuddanda
}

Received: 21 December 2008 / Accepted: 3 February 2009/Published online: 17 March 2009

(C) Springer-Verlag London Ltd 2009

A 35-year-old man presented with progressive exertional dyspnea (3 months) and syncopal attacks (1 week). He had tachypnea, tachycardia and hypotension. Examination revealed a mid-diastolic plop at the apex. Clinical suspicion was a left atrial mass with a ball valve-like mechanism (LA thrombus or LA tumors). Echo and cardiac MRIs with gadolinium enhancement (to differentiate between a tumor and thrombus) were considered as necessary investigations. An emergency echo revealed a large mass $(8.0 \times 3.5 \mathrm{~cm})$ in the left atrium arising from the roof. It was prolapsing through the mitral valve, compromising LV inflow. There were no embolic manifestations or features of NAME (nevi, atrial myxoma, myxoid neurofibromas, ephelides) or LAMB (lentigenes, atrial myxoma, blue nevi) syndromes or Carney's complex. The patient underwent surgery. The excised mass was conical, and histopathology revealed myxomatous areas with vascular channels and cellular areas. This was diagnosed as a cellular variant of angiomyxoma (a rare variant). The patient recovered with no complications and was discharged after 1 week of obser-

G. Sogunuru

Kishanvuddanda, S/o V. Dhananjaya, 13-07-939 G/A, Korlagunta, Tirupathi,

Chittoor district, Andhra Pradesh, India

K. Vuddanda $(\bowtie)$

Kishanvuddanda, C/O DBR hospitals, Mosque road, Tirupathi, Chittoor district, Andhra Pradesh, India

e-mail: Kishanvuddanda@gmail.com vation. Atrial myxoma is the most common benign primary tumor of the heart. The majority of them are solitary and occur in the left atrium in the zone of the fossa ovalis. Common symptoms are dyspnea and syncope. Other presentations include PUO, weight loss, embolic manifestations and arrhythmias. Definitive treatment is always surgery. The present case emphasizes the clinical significance of cardiac auscultation in a patient who presents with syncope.



Fig. 1 Echo (apical 4 chamber view) showing a large left atrial mass prolapsing through the mitral orifice 


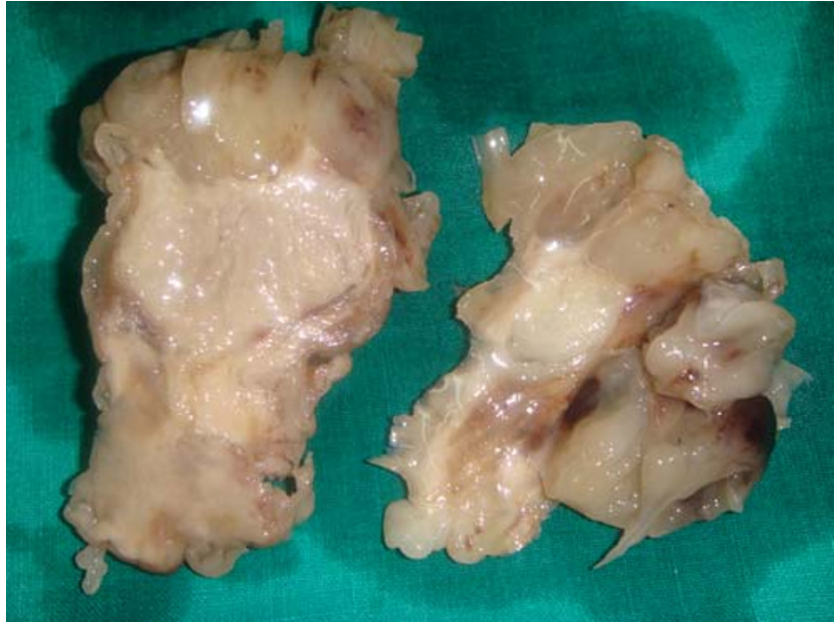

Fig. 2 Cut section of resected LA mass showing the glistening central myxomatous areas

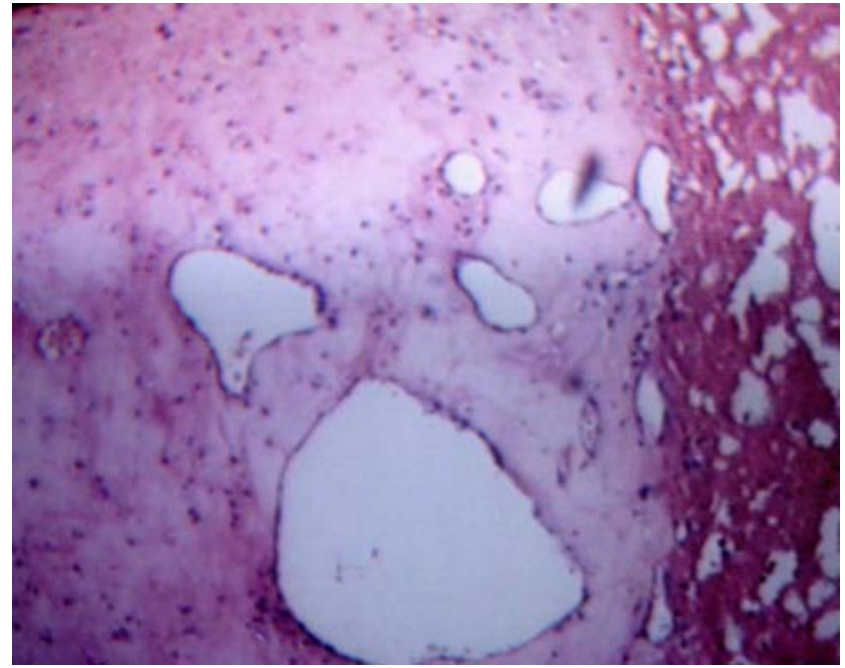

Fig. 3 LA mass histopathology (H \& E) showing vascular channels in the centre along with cellular areas consistent with cellular variant of angiomyxoma 